\title{
SPECTROSCOPY OF OLD NOVA CANDIDATES
}

\author{
L. Schmidtobreick, ${ }^{1}$ C. Tappert,${ }^{2}$ A. Bianchini, ${ }^{3}$ and R. E. Mennickent ${ }^{2}$
}

We present optical spectroscopy for several old nova candidates that hitherto lacked such observations. V842 Cen, V630 Sgr, XX Tau, CQ Vel, and V840 Oph show typical CV spectra, but V529 Ori is probably misclassified.

V630 Sgr is an eclipsing system with an orbital period of $2.83 \mathrm{~h}$. In spite of the high inclination, the emission lines are not double-peaked at the resolution of $1 \mathrm{~nm}$.

V840 Oph's spectrum confirms the identification. The strength of C IV and similarities to QU Car (Drew et al. 2003) suggest a carbon-star secondary. The highly inverse Balmer decrement and the highexcitation lines point towards high temperatures and a magnetic system (Schnidtobreick et al. 2003)

XX Tau's recovery by Cohen (1985) is confirmed by the spectrum. The presence of the Balmer series down to H 11, and the strength of HeI compared to He II resembles more a dwarf nova than a classical nova. It might thus be that XX Tau represents ac-

\footnotetext{
${ }^{1}$ Europ. South. Obs., Casilla 19001, Santiago 19, Chile.

${ }^{2}$ Grupo de Astronomía, Universidad de Concepción, Casilla 160-C, Concepción, Chile.

${ }^{3}$ Dipartimento di Astronomia, Università di Padova, Vicolo dell'Osservatorio 2, I-35122, Padova, Italy.
}

tually a link between these two CV populations - a low mass transfer nova.

V842 Cen (Fig. 1) has been discovered by McNaught in 1986 (IAUC 4274). The spectrum is similar to that of XX Tau indicating a short orbital period and an accordingly low mass transfer rate. The comparison of the line profiles shows that $\mathrm{H} \alpha$ is still strongly disturbed by N II which indicates that V842 Cen has not yet reached its quiescence level.

V529 Ori's spectrum shows a strong but narrow $\mathrm{H} \alpha$ line. No other emission lines are visible and the continuum is extremely red, letting a nova classification appear doubtful.

CQ Vel's spectrum shows the Balmer lines and He II in emission and thus confirms the identification by Woudt \& Warner (2001).

\section{REFERENCES}

Cohen, J.G. 1985, ApJ 292, 90

Downes R.A. et al. 2001, PASP 113, 764, living edition Drew, J.E., Hartley, L.E., Long, K.S., \& van der Walt, J. 2003, MNRAS 338, 401

Schmidtobreick, L., Tappert, C., Bianchini, A., \& Mennickent, R.E. 2003, A\&A, in press

Woudt, P.A. \& Warner, B. 2001, MNRAS 328, 159

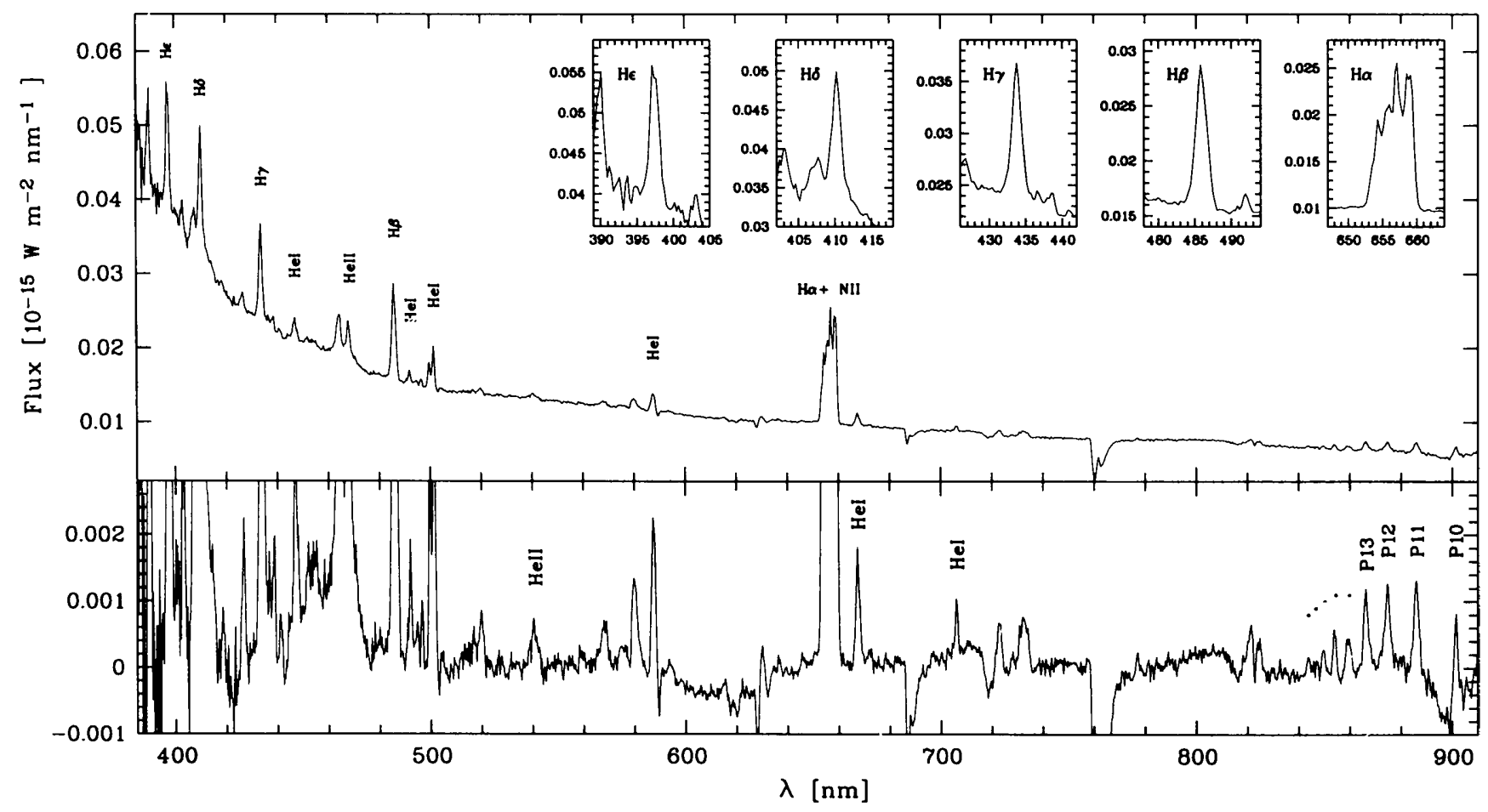

Fig. 1. As example we show the spectrum of V842 Cen. 